\title{
SERUM GLP-2 is Increased in Association with Excess Gestational Weight Gain
}

\author{
Maike K. Kahr, MD ${ }^{1,2}$ Kathleen M. Antony, MD ${ }^{1,3}$ Megan Galindo, MD ${ }^{1}$ Megan Whitham, MD \\ Min Hu, MD ${ }^{1}$ Kjersti M. Aagaard, MD, PhD ${ }^{1}$ Melissa A. Suter, PhD ${ }^{1}$ \\ ${ }^{1}$ Department of Obstetrics and Gynecology, Baylor College of \\ Medicine, Houston, Texas \\ 2 Department of Obstetrics and Gynecology, University Hospital \\ Zurich, Zurich, Switzerland \\ ${ }^{3}$ Department of Obstetrics and Gynecology, University of Wisconsin- \\ Madison, Madison, Wisconsin \\ Am J Perinatol 2023;40:400-406. \\ Address for correspondence Melissa Suter, PhD, Department of \\ Obstetrics and Gynecology, Baylor College of Medicine, 1 Baylor Plaza, \\ Rm. 314C, Houston, TX 77030 (e-mail: masuter@bcm.edu).
}

\section{Abstract \\ Keywords \\ - gestational weight gain \\ - gestational diabetes \\ - obesity \\ - metabolic endotoxemia}

Objective Obesity in pregnancy bears unique maternal and fetal risks. Obesity has also been associated with chronic inflammation, including elevated serum levels of interleukin-6 (IL-6) and tumor necrosis factor- $\alpha$ (TNF- $\alpha$ ). Higher serum lipopolysaccharide (LPS) levels have been implicated in driving this inflammation, a phenomenon called metabolic endotoxemia (ME). GLP-2, a proglucagon-derived peptide, is believed to be integral in maintaining the integrity of the intestine in the face of LPS-mediated endotoxemia. We hypothesized that obesity and/or excess weight gain in pregnancy would be associated with an increase in maternal and neonatal markers of ME, as well as GLP-2.

Study Design Paired maternal and neonatal (cord blood) serum samples $(n=159)$ were obtained from our pregnancy biobank repository. Serum levels of LPS, endotoxin core antibody-immunoglobulin M (EndoCAb-lgM), and GLP-2 were measured by ELISA. IL-6 and TNF- $\alpha$ were measured using a Milliplex assay. Results were stratified by maternal body mass index (BMI), maternal diabetes, and gestational weight gain (GWG).

Results Maternal IL-6 is significantly decreased in the obese, diabetic cohort compared with the nonobese, nondiabetic cohorts (95.28 vs. $99.48 \mathrm{pg} / \mathrm{mL}, p=0.047$ ), whereas GLP-2 is significantly increased ( 1.92 vs. $2.89 \mathrm{ng} / \mathrm{mL}, p=0.026$ ). Neonatal TNF$\alpha$ is significantly decreased in the obese cohort compared with the nonobese cohort ( 12.43 vs. $13.93 \mathrm{pg} / \mathrm{mL}, p=0.044$ ). Maternal GLP-2 is significantly increased in women with excess GWG compared with those with normal GWG $(2.27$ vs. $1.48 \mathrm{ng} / \mathrm{mL}$, $p=0.014)$. We further found that neonatal IL- 6 and TNF- $\alpha$ are negatively correlated with maternal BMI $(-0.186, p=0.036$ and $-0.179, p=0.044$, respectively) and that maternal and neonatal IL-6 showed a positive correlation $(0.348, p<0.001)$.

Conclusion Although we observed altered levels of markers of inflammation (IL-6 and TNF- $\alpha$ ) with maternal obesity and diabetes, no changes in LPS or endoCAb-IgM were observed. We hypothesize that the increased GLP-2 levels in maternal serum in association with excess GWG may protect against ME in pregnancy. received

June 10, 2020 accepted after revision March 2, 2021 article published online May 3, 2021
DOI https://doi.org/ $10.1055 / \mathrm{s}-0041-1728828$ ISSN 0735-1631.

\footnotetext{
(C) 2021. The Author(s).

This is an open access article published by Thieme under the terms of the Creative Commons Attribution-NonDerivative-NonCommercial-License, permitting copying and reproduction so long as the original work is given appropriate credit. Contents may not be used for commercial purposes, or adapted, remixed, transformed or built upon. (https://creativecommons.org/ licenses/by-nc-nd/4.0/) Thieme Medical Publishers, Inc., 333 Seventh Avenue, 18th Floor, New York, NY 10001, USA
} 


\section{Key Points}

- Maternal serum levels of GLP-2, a proglucagon-derived peptide, are increased in obese, diabetic gravidae.

- Maternal serum GLP-2 levels are also increased in association with excess gestational weight gain compared with normal gestational weight gain.

- GLP-2 may be increased in association with obesity and weight gain to protect against metabolic endotoxemia in pregnancy.

Whereas obesity has long been known as a risk factor for metabolic syndrome (MetS), a complex disorder characterized by an increased risk of cardiovascular disease and type 2 diabetes, ${ }^{1}$ not all obese individuals develop MetS. A strong predictor of MetS susceptibility includes elevated levels of markers of inflammation such as interleukin-6 (IL-6) and tumor necrosis factor- $\alpha$ (TNF- $\alpha)$ in obese individuals., ${ }^{2,3}$ Whereas the etiology of these elevated inflammatory markers is unknown, one explanation suggests that bacteria-derived compounds are able to translocate across the gut barrier (i.e., "leaky gut") in association with obesity, driving this increase in inflammation. ${ }^{4,5}$ This phenomenon has been termed metabolic endotoxemia (ME).

A hallmark of ME is elevated serum levels of lipopolysaccharide (LPS), the main component of the cell wall of gramnegative bacteria. ${ }^{6,7} \mathrm{ME}$ has been shown in mouse models to be induced by high fat diet feeding. ${ }^{5}$ ME has also been associated with a decrease in the anti-endotoxin core antibody-immunoglobulin $\mathrm{M}$ (endoCAb-IgM) in obese, diabetic women. ${ }^{8}$ GLP-2, a gut-derived glucagon-like peptide, enhances gut barrier function, ${ }^{9}$ therefore reducing gut permeability, and may protect against a leaky gut and the subsequent systemic markers of endotoxemia. ${ }^{10,11}$

To support the metabolic demands of a growing fetus, pregnancy is characterized by relative maternal insulin resistance. ${ }^{12,13}$ Insulin resistance arises as the result of cumulative effects of several key physiologic changes of pregnancy designed to supply glucose to the fetus and the placenta. ${ }^{13}$ It is thought that maternal obesity is associated with further increased insulin resistance, manifesting clinically as a higher occurrence of gestational diabetes mellitus (GDM) among obese pregnant women. In turn, GDM predisposes these same women to postpregnancy MetS and type 2 diabetes among others. ${ }^{14-16}$ Similarly, excess gestational weight gain is an independent risk factor for maternal and fetal metabolic perturbations. ${ }^{17}$ Others have shown that pregestational obesity is associated with the ME markers of increased serum LPS and IL-6 levels in pregnancy ${ }^{18}$ revealing that ME may be an increased risk for maternal and fetal health. According to the developmental origins of health and disease (DOHaD), exposure to an adverse in utero environment is associated with an increased risk of MetS in adulthood. ${ }^{19,20}$ Whereas many factors likely contribute to this increased risk, exposure to maternal ME in utero may set the stage for metabolic perturbations in adulthood.

In this study, we sought to determine if obese and/or diabetic mothers have altered circulating levels of $\mathrm{ME}$ markers (IL-6, TNF- $\alpha$, LPS, and EndoCAb) compared with women with recommended weight. We also sought to determine if the offspring of obese, diabetic mothers would have markers of ME. Further, we measured the levels of maternal and neonatal GLP-2 to determine if levels are altered with obesity, diabetes, or gestational weight gain.

\section{Materials and Methods}

Study subjects were recruited by skilled research personnel after obtaining full and informed consent from eligible pregnant women after admission to labor and delivery as we have previously described (IRB no.: H-26364). ${ }^{21-25}$ Maternal and neonatal (cord blood) serum samples were obtained from the specimen repository, PeriBank, and $>4,900$ clinical variables were available for subjects, which had been extracted from electronic medical records, alongside in-person interviews. For this study, extracted data include maternal age, prepregnancy body mass index (BMI), BMI at delivery, gestational age at delivery, birth weight, and maternal diabetes status. We included both type 2 (i.e., preexisting diabetes) and gestational diabetes for this study. None of our patients exhibited type 1 diabetes. Neonatal (cord) blood specimens were collected directly after delivery and maternal blood specimens within 8 hours postpartum. Samples were stored at $-80^{\circ} \mathrm{C}$ until use. Exclusion criteria included gestational age $<32$ weeks, multiple gestations, or known fetal anomalies.

We utilized paired maternal and neonatal (cord) serum samples ( $n=159$ maternal and $n=159$ neonatal) from PeriBank to analyze levels of serum LPS, EndoCAb-IgM, TNF- $\alpha$, IL-6, and GLP-2. Commercially available ELISA assays were utilized to measure LPS (Thermo Scientific, Rockford, Illinois, United States), EndoCAb-IgM (Hycult Biotech, Uden, Netherlands), and GLP-2 (Merck Millipore, St. Charles, Missouri, United States) in paired maternal and neonatal serum samples. A standard curve was created for each plate using standards provided by the manufacturer. TNF- $\alpha$ and IL-6 levels were measured using the Human Metabolic Magnetic Bead Panel (kit \# HMHEMAG34k, EMD Millipore, Billerica, Massachusetts, United States) in a MagPix system (Luminex Corporation, Austin, Texas, United States) through our Digestive Disease Center core facility. Raw data were acquired using XPONENT for Magpic (version 4.2) and analyzed with Milliplex Analyst (version 5.1.0.0 standard). All samples were performed in duplicate.

\section{Statistical Analysis}

Statistical analyses and data processing were performed with SPSS (version 24, IBM, Armonk, NY). Descriptive statistics were used to characterize demographic variables. Data are given as median [interquartile range]. Sample size was chosen based on 


\begin{tabular}{|c|c|c|c|c|c|}
\hline & \multirow[t]{2}{*}{ Nondiabetic $(n=112)$} & \multicolumn{4}{|l|}{ Diabetic $(n=47)$} \\
\hline & & Type $2(n=8)$ & GDM A1 $(n=11)$ & GDM A2 $(n=28)$ & $\begin{array}{l}\text { Any diabetes } \\
(n=47)\end{array}$ \\
\hline Age $(y)$ & $27.4[22.6 ; 32.2]$ & $31.1[23.6 ; 37.2]$ & 31.7 [25.6; 35.7] & $30.4[27.3 ; 35.4]^{* *}$ & $30.8[26.2 ; 35.6]^{* *}$ \\
\hline $\begin{array}{l}\text { BMI (third } \\
\text { trimester, } \\
\mathrm{kg} / \mathrm{m}^{2} \text { ) }\end{array}$ & $29.7[26.8 ; 33.7]$ & $33.2[28.6 ; 37.6]$ & $33.8[28.4 ; 40.1]$ & $32.5[28.7 ; 37.5]^{*}$ & $32.9[28.8 ; 37.5]^{* *}$ \\
\hline $\begin{array}{l}\text { Gestational } \\
\text { age (wk) }\end{array}$ & $39.2[38.2 ; 40.1]$ & $36.8[35.3 ; 38.1]^{* *}$ & $39.0[36.4 ; 41.0]$ & $37.6[36.9 ; 39.1]^{* *}$ & $37.6[36.7 ; 39.1]^{* * *}$ \\
\hline $\begin{array}{l}\text { Birth } \\
\text { weight }(g)\end{array}$ & $\begin{array}{l}3249.0 \\
{[2940.0 ; 3551.5]}\end{array}$ & $\begin{array}{l}3160 \\
{[2777.5 ; 3588.8]}\end{array}$ & $\begin{array}{l}3170 \\
{[2829.0 ; 3800.0]}\end{array}$ & $\begin{array}{l}3254.5 \\
{[2766.3 ; 3471.3]}\end{array}$ & $\begin{array}{l}3244.0 \\
{[2785.5 ; 3525.0]}\end{array}$ \\
\hline
\end{tabular}

Abbreviations: BMI, body mass index; GDM, gestational diabetes mellitus.

Note: Maternal age, BMI, and gestational age at delivery significantly differed between the nondiabetic and diabetic cohorts in our study population. Statistically significant differences in comparison to non-diabetic women are marked by asterisks ${ }^{*} p<0.05,{ }^{* *} p<0.01,{ }^{* * *} p<0.001$.

the assumption of a $10 \%$ difference between groups, with a type 1 error rate of $5 \%$ and power of 0.8 . Shapiro-Wilk and Kolmogorov-Smirnov tests revealed that our data were not normally distributed. Therefore, Spearman's rank correlation was utilized to determine the reported correlations. Patients were stratified by the third trimester BMI into nonobese (BMI $<30.0$ ) or obese (BMI $\geq 30.0$ ) cohorts. We also stratified based on maternal diabetes (nondiabetic and diabetic), as well as based on diabetic classification (type 2, GDMA1, and GDMA2) and on gestational weight gain. ${ }^{26}$ Subjects were classified as type 2 diabetic if they had been diagnosed before pregnancy, according to medical records. Gestational diabetes was diagnosed using clinical criteria, specifically using accepted Carpenter-Coustan glucose tolerance test cutoffs ( $\geq 140 \mathrm{mg} / \mathrm{dL}){ }^{27}$ In accordance with established classification schemes, GDMA1 included subjects who maintained euglycemia with nutritional therapy, whereas those classified as GDMA2 required antihyperglycemic agents or insulin as has been described. ${ }^{28}$ Mann-Whitney and Kruskal-Wallis tests were performed to analyze differences between BMI/diabetes groups and serum parameters. Correlations between maternal GLP-2 and other measured maternal metabolites were determined using Spearman's rho. A $p$-value of $<0.05$ was accepted as significant throughout the study.

\section{Results}

In total, serum samples from 318 subjects were utilized in this study (159 maternal-neonatal dyads). There was insufficient sample amount to measure each and every marker for both maternal and neonatal samples; therefore, we have indicated the number of samples for each metabolite in each figure for every category. Subjects were stratified either according to the third trimester BMI as nonobese $(\mathrm{BMI}<30.0)$ or obese (BMI $\geq 30.0$ ). In our cohort, 47 women were diabetic. Maternal age, gestational age at delivery, and maternal BMI significantly differed between groups ( $p=0.01$; - Table 1$)$.

We investigated the contribution of obesity to altered markers of ME. Comparing nonobese to obese individuals, regardless of diabetic diagnosis, we did not observe any changes in maternal levels of any marker measured (-Table 2; -Fig. 1A). However, we did find a significant decrease in TNF- $\alpha$ in the offspring of obese subjects compared with the nonobese (-Table 2; -Fig. 1B). We further sought to determine if maternal or neonatal markers corresponded with maternal BMI. Whereas we did not find any significant correlations between maternal serum markers and maternal BMI, neonatal IL- 6 and TNF- $\alpha$ were negatively correlated with maternal BMI $(-0.186, p=0.036$ and $-0.179, p=0.044$, respectively; - Table 3 ).

\begin{tabular}{|c|c|c|c|c|}
\hline & \multicolumn{2}{|c|}{ Nonobese } & \multicolumn{2}{|c|}{ Obese } \\
\hline & Maternal & Neonatal & Maternal & Neonatal \\
\hline LPS (EU/mL) & $0.51[0.41 ; 0.67]$ & $1.01[0.85 ; 1.23]$ & $0.54[0.40 ; 0.73]$ & $0.90[0.67 ; 1.20]$ \\
\hline endoCAb-IgM (MMU/mL) & $96.35[89.10 ; 103.40]$ & $24.40[20.60 ; 32.80]$ & $94.88[88.63 ; 101.69]$ & $27.40[22.05 ; 39.58]$ \\
\hline IL-6 (pg/mL) & $71.65[30.14 ; 131.44]$ & $24.28[10.09 ; 64.78]$ & $54.81[24.14 ; 124.44]$ & $18.02[6.81 ; 36.64]$ \\
\hline TNF- $\alpha(p g / m L)$ & $6.41[4.37 ; 9.30]$ & $13.71[11.27 ; 17.33]$ & $7.22[4.71 ; 10.06]$ & $11.81[8.76 ; 15.28]^{a}$ \\
\hline GLP-2 (ng/mL) & $1.50[0.75 ; 2.47]$ & $1.40[0.72 ; 2.35]$ & $1.49[1.14 ; 2.22]$ & $1.74[1.10 ; 2.67]$ \\
\hline
\end{tabular}

Abbreviations: EndoCab-IgM, endotoxin core antibodies-immunoglobulin M; GLP-2, glucagon-like peptide-2; IL-6, interleukin-6; LPS, lipopolysaccharides; TNF- $\alpha$, tumor necrosis factor- $\alpha$.

Note: Data are given as median [interquartile range].

astatistically significant differences between groups, with $p<0.05$. 

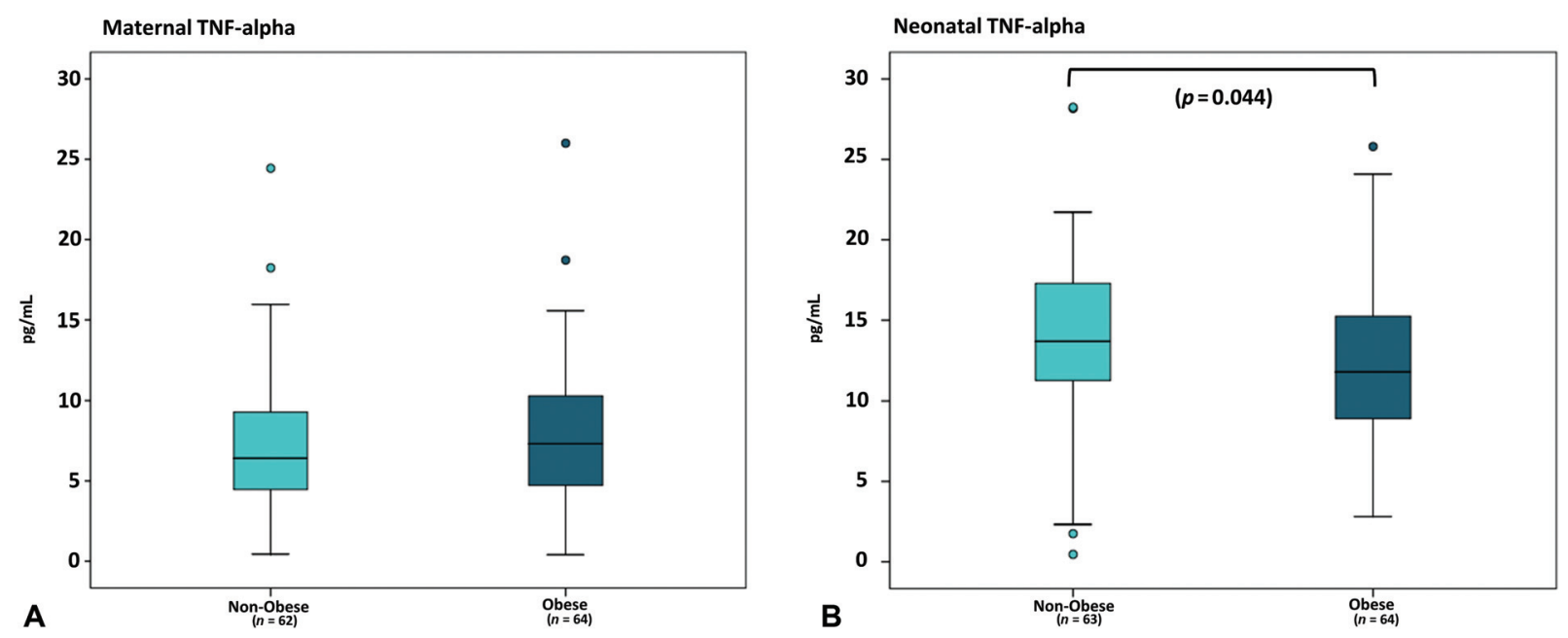

Fig. 1 Neonatal tumor necrosis factor- $\alpha$ (TNF- $\alpha$ ) is significantly decreased in association with maternal obesity. (A) Whereas maternal serum TNF- $\alpha$ is not altered between nonobese and obese subjects, (B) neonatal levels are decreased in the obese cohort compared with nonobese cohorts.

Table 3 Correlation between metabolic endotoxemia (ME) markers and maternal body mass index (BMI) at delivery

\begin{tabular}{|lll|} 
& \multicolumn{1}{c|}{ Maternal } & \multicolumn{2}{c|}{$\begin{array}{c}\text { Neonatal } \\
\text { Spearman's rho }\end{array}$} \\
LPS (EU/mL) & $0.007, p=0.949$ & $-0.036, p=0.735$ \\
endoCAb-lgM & $-0.197, p=0.065$ & $0.174, p=0.103$ \\
$(\mathrm{MMU} / \mathrm{mL})$ & & \\
IL-6 $(\mathrm{pg} / \mathrm{mL})$ & $-0.081, p=0.370$ & $-0.186, p=0.036^{\mathrm{a}}$ \\
TNF- $\alpha(\mathrm{pg} / \mathrm{mL})$ & $0.125, p=0.162$ & $-0.179, p=0.044^{\mathrm{a}}$ \\
$\mathrm{GLP}-2(\mathrm{ng} / \mathrm{mL})$ & $0.120, p=0.304$ & $0.148, p=0.228$ \\
\hline
\end{tabular}

Abbreviations: EndoCab-IgM, endotoxin core antibodies-immunoglobulin M; GLP-2, glucagon-like peptide-2; IL-6, interleukin-6; LPS, lipopolysaccharides; TNF- $\alpha$, tumor necrosis factor- $\alpha$.

${ }^{a}$ Statistically significant correlations: $p<0.05$.

We also sought to stratify our cohort by gestational weight gain (insufficient, recommended, and excess) ${ }^{26}$ for the subjects for which we had the first trimester weight information available. We found that although most markers were not affected by gestational weight gain ( - Table 4), GLP-2 was significantly increased in maternal serum in subjects with excessive gestational weight gain compared with those with recommended weight gain, but not in neonatal (-Fig. 2).

Because the samples were paired such that every maternal sample was run in parallel with the corresponding neonatal sample, we wanted to determine if there were any correlations between maternal and neonatal levels for any of the markers measured. We found that only IL-6 levels were significantly, positively correlated $(0.355, p<0.001$; - Table 5$)$. No correlations were found for other markers. We also sought to determine if maternal GLP-2 was correlated with any other maternal serum cytokine level. We did not find any significant correlations between GLP-2 and other metabolic markers (data not shown).

Because ME has been associated with obesity and diabetes, ${ }^{8}$ we measured the markers of ME stratified by diabetes in our analysis. Previous work in nonpregnant women revealed that LPS was increased and EndoCAbIgM decreased in obese nonpregnant women with diabetes,

Table 4 Average levels of measured serum markers in subjects of different gestational weight gain categories

\begin{tabular}{|c|c|c|c|c|c|c|}
\hline & \multicolumn{2}{|l|}{ Insufficient } & \multicolumn{2}{|l|}{ Normal } & \multicolumn{2}{|l|}{ Excess } \\
\hline & Maternal & Neonatal & Maternal & Neonatal & Maternal & Neonatal \\
\hline LPS (EU/mL) & $0.46[0.38 ; 0.78]$ & $0.89[0.66 ; 1.19]$ & $0.55[0.43 ; 0.69]$ & $1.02[0.45 ; 1.21]$ & $0.52[0.37 ; 0.73]$ & $0.93[0.78 ; 1.27]$ \\
\hline $\begin{array}{l}\text { endoCAb-lgM } \\
(\mathrm{MMU} / \mathrm{mL})\end{array}$ & $\begin{array}{l}26.95 \\
{[22.78 ; 33.40]}\end{array}$ & $\begin{array}{l}26.95 \\
{[22.78 ; 33.40]}\end{array}$ & $\begin{array}{l}97.50 \\
{[87.38 ; 103.16]}\end{array}$ & $\begin{array}{l}27.58 \\
{[22.76 ; 33.51]}\end{array}$ & $\begin{array}{l}93.25 \\
{[89.43 ; 100.65]}\end{array}$ & $\begin{array}{l}30.40 \\
{[23.00 ; 40.90]}\end{array}$ \\
\hline IL-6 (pg/mL) & $\begin{array}{l}44.60 \\
{[21.38 ; 106.08]}\end{array}$ & $\begin{array}{l}25.28 \\
{[9.73 ; 52.25]}\end{array}$ & $\begin{array}{l}74.59 \\
{[34.25 ; 131.75]}\end{array}$ & $\begin{array}{l}10.53 \\
{[6.79 ; 58.53]}\end{array}$ & $\begin{array}{l}62.80 \\
{[31.65 ; 123.44]}\end{array}$ & $\begin{array}{l}14.70 \\
{[6.64 ; 38.15]}\end{array}$ \\
\hline $\begin{array}{l}\text { TNF- } \alpha \\
(\mathrm{pg} / \mathrm{mL})\end{array}$ & $6.10[4.34 ; 9.31]$ & $\begin{array}{l}12.21 \\
{[9.98 ; 16.09]}\end{array}$ & 7.04 [4.99; 8.33] & $11.93[9.81 ; 15.38]$ & $7.54[4.56 ; 8.96]$ & $13.95[9.38 ; 16.53]$ \\
\hline $\begin{array}{l}\text { GLP-2 } \\
\text { (ng/mL) }\end{array}$ & $1.34[1.13 ; 1.99]$ & $1.16[0.78 ; 2.23]$ & $1.24[0.87 ; 1.73]$ & $1.53[0.92 ; 2.90]$ & $1.88[1.51 ; 3.70]^{\mathrm{a}}$ & $1.79[1.32 ; 3.30]$ \\
\hline
\end{tabular}

Abbreviations: EndoCab-IgM, endotoxin core antibodies-immunoglobulin M; GLP-2, glucagon-like peptide-2; IL-6, interleukin-6; LPS, lipopolysaccharides; TNF- $\alpha$, tumor necrosis factor- $\alpha$.

Note: Average levels of measured serum markers in subjects of different gestational weight gain categories.

${ }^{\mathrm{a} S}$ Statistically significant differences in comparison to subjects with normal weight gain. $p<0.05$. 

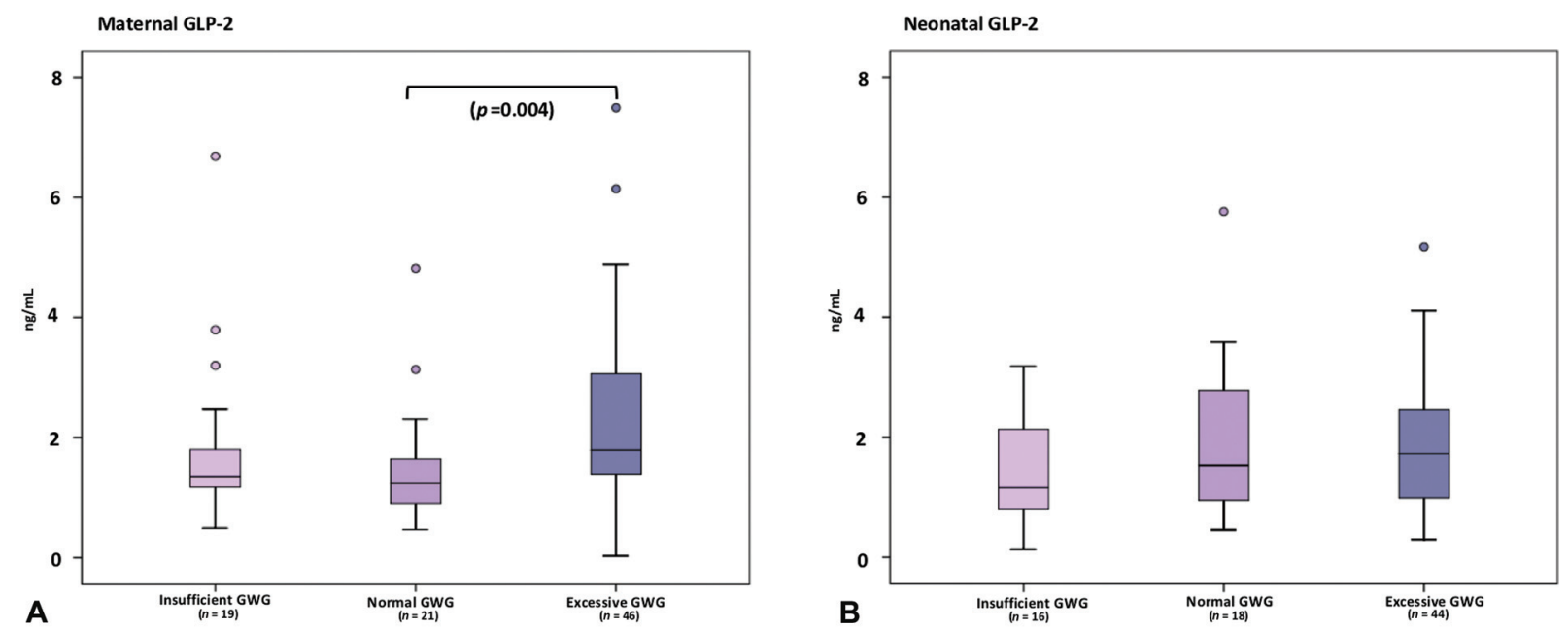

Fig. 2 Maternal glucagon-like peptide-2 (GLP-2) levels are significantly increased with excessive gestational weight gain (GWG). (A) Compared with subjects with normal gestational weight gain, those with excessive weight gain in pregnancy have elevated levels of GLP-2. (B) No differences are observed in the neonates in GLP-2 serum levels.

Table 5 Maternal and neonatal IL-6 levels are significantly correlated

\begin{tabular}{ll|}
\hline LPS & Spearman's rho \\
EndoCAb-lgM & $-0.134, p=0.187$ \\
IL-6 & $-0.100, p=0.328$ \\
TNF- $\alpha$ & $0.355, p<0.001^{\text {a }}$ \\
GLP-2 & $0.148, p=0.087$ \\
\hline
\end{tabular}

Abbreviations: EndoCab-IgM, endotoxin core antibodies-immunoglobulin M; GLP-2, glucagon-like peptide-2; IL-6, interleukin-6; LPS, lipopolysaccharides; TNF- $\alpha$, tumor necrosis factor- $\alpha$.

aStatistically significant correlation.

but not in obese women without diabetes. ${ }^{8}$ We did not find any significant changes in LPS levels or EndoCAb-IgM in either the maternal or neonatal serum when stratifying by diabetes (- Supplementary Table S1, available in the online version). We also measured the inflammatory markers TNF- $\alpha$ and IL- 6 in maternal and neonatal samples. IL-6 was significantly reduced in the obese diabetic mothers compared with the nonobese, nondiabetic controls ( - Fig. 3A). No differences were observed in the matching neonatal samples ( - Fig. 3B). We also measured levels of TNF- $\alpha$. Our findings reveal that TNF- $\alpha$ is increased in both obese cohorts (nondiabetics and diabetics) compared with the nonobese, nondiabetic group ( - Fig. $3 \mathrm{C}$ ). There was no change in the neonatal serum ( - Fig. 3D).

\section{Discussion}

Although ME has been characterized in nonpregnant women, few studies focus on its importance to the mother and the fetus in pregnancy. ${ }^{18,29,30}$ Given insights from the DOHaD hypothesis, individuals exposed in utero to ME may be at risk of development of metabolic perturbations later in life. However, consideration must be given to what markers of ME are able to cross the placenta from maternal to fetal circulation, and what markers may be arising from the placental or fetal response.

Placental perfusion studies using term, human placentae, revealed that little to no IL- 6 or TNF- $\alpha$ can cross the placenta. ${ }^{31}$ This is important to note, as we observed a correlation between maternal and neonatal serum levels ( - Table 3 ). Furthermore, we found that both neonatal IL- 6 and TNF- $\alpha$ levels were inversely correlated with maternal BMI at delivery (- Table 2). Because these cytokines do not cross the placenta, it is likely that they are derived from the neonatal response to the in utero environment.

It is known that whereas maternal IgG readily crosses the placenta, transfer of IgM is rare. ${ }^{32}$ Although we did not find significant differences in maternal or neonatal endoCAb-IgM, there were detectable amounts of endoCAb-IgM in the neonatal serum ( - Tables 2 and 4 ). Similarly, there were also detectable levels of LPS in neonatal serum ( - Tables 2 and 4 ). Therefore, we conclude that the fetus was exposed to LPS and was able to give an immunological response.

The idea that the developing fetus is exposed to bacteria is not a new one. We and others have shown that the in utero environment is not sterile. In fact, we have reported that the placenta harbors a unique microbiome that changes in association with preterm birth and gestational weight gain. ${ }^{33-35}$ Our current study reveals, through detection of endoCAb-IgM in the cord blood, that such in utero exposures are likely to be involved in development of the fetal immune system.

Strengths of this study include the availability of matching pairs of maternal and neonatal serum samples for included study subjects. While utilizing the expertise of our laboratory as well as of a specialized core facility to analyze core endotoxemia markers, the insufficient amount of maternal and neonatal serum resulting in diminished completeness of some endotoxemia markers poses a limitation of this study. Another limitation includes the single time point of serum collection for both mother and neonate (at delivery), as these markers may change rapidly postpartum. Further limitations of this study 

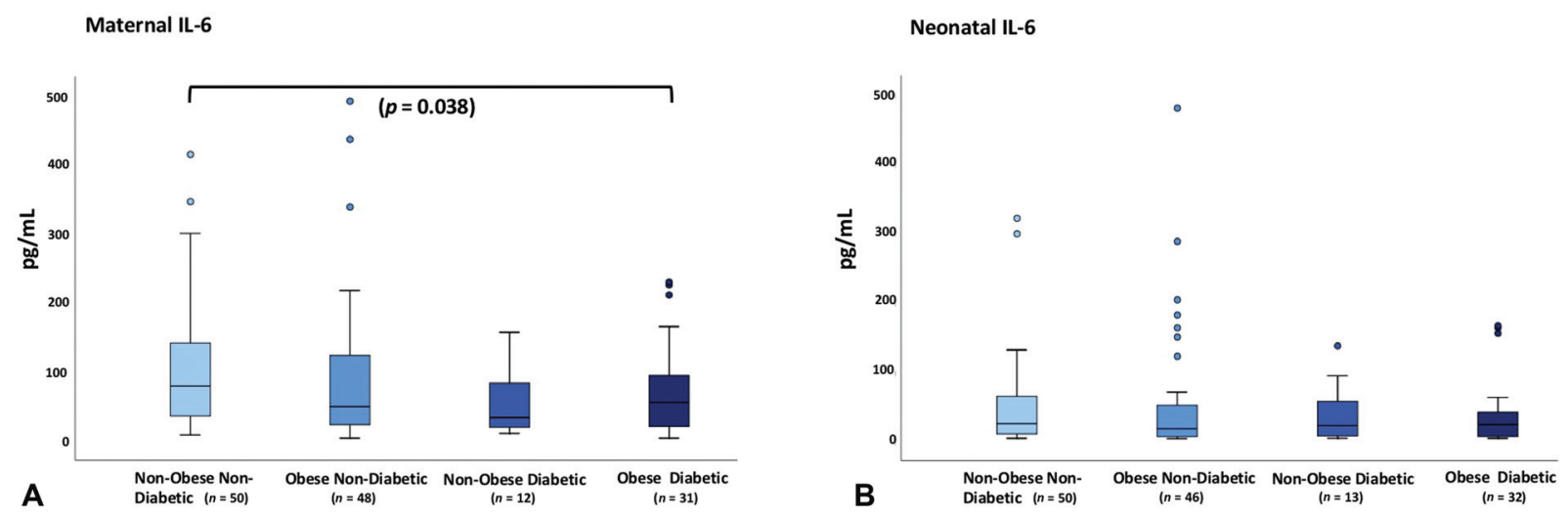

Maternal TNF-alpha

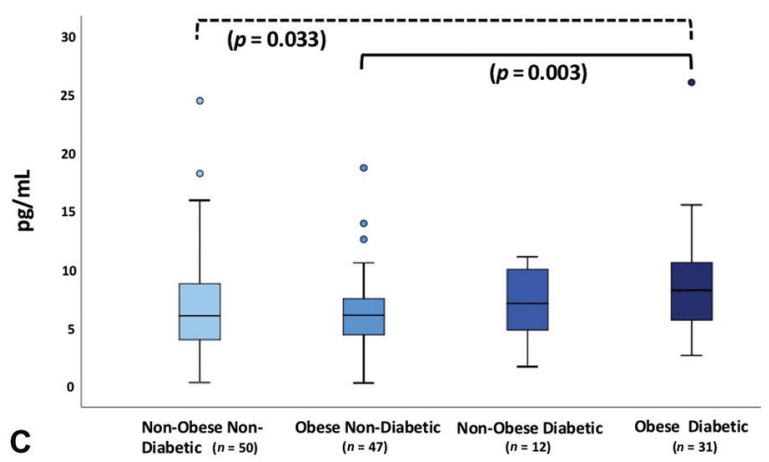

Fetal TNF-alpha

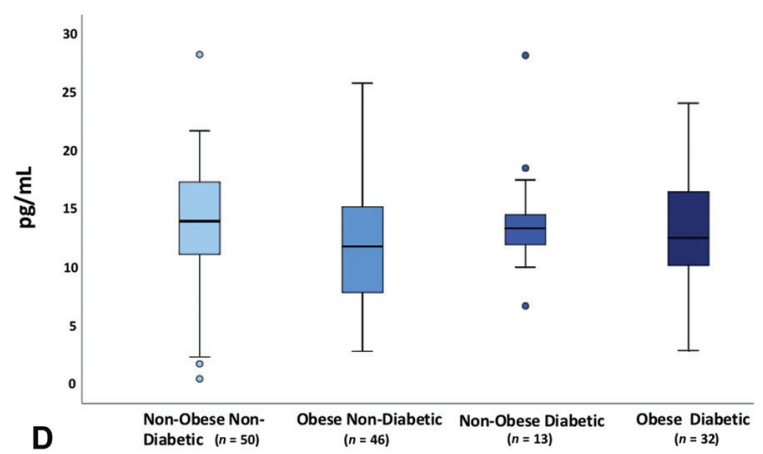

Fig. 3 Maternal interleukin-6 (IL-6) and tumor necrosis factor- $\alpha$ (TNF- $\alpha$ ) levels are altered in association with maternal diabetes. (A) IL-6 is significantly reduced in obese, diabetic mothers compared with the nonobese, nondiabetic controls, (B) whereas no differences are seen in the neonates. (C) Maternal TNF- $\alpha$ is significantly increased in the obese, diabetic subjects compared with the nonobese, nondiabetic subjects, (D) whereas no changes are observed in the neonates.

include the lack of information on the treatment of a present GDM or type 2 diabetes mellitus. Since a subset of gravidae began their prenatal care at our center toward the end of pregnancy, we lack complete information on prepregnancy and the first trimester BMI and consequently on GWG for such individuals. Additionally, further research should also focus on the maternal long-term outcomes, meaning the predisposition toward type 2 diabetes mellitus, as well as the long-term outcomes of neonates in relation to altered ME markers.

We hypothesized that maternal obesity, diabetes, and excess gestational weight gain would be associated with $\mathrm{ME}$ in the mother and therefore provide an adverse in utero environment for the developing fetus. However, we did not detect significant changes to either LPS or endoCAb-IgM in maternal serum, which are hallmarks of ME. This could be partially explained by the changes in the levels of GLP-2 we found with gestational weight gain ( - Fig. 2). Perhaps this is protective against increased markers of ME in the maternal serum during pregnancy. To our knowledge, only one other study has addressed intestinal permeability in overweight pregnant women with regard to ME. ${ }^{30}$ That study measured maternal serum levels of zonulin, a protein that modulates intestinal permeability. They found that zonulin and increased levels of LPS were associated positively. Therefore, follow-up studies are necessary to address this discrepancy, measuring GLP-2, zonulin, and LPS levels with respect to maternal BMI, gestational weight gain, and maternal diabetes.

Funding

This work was funded by a pilot award from the Texas Medical Center Digestive Disease Center (P30DK56338 to M.A.S.), a grant from the U.S. Department of Health and Human Services, National Institutes of Health NIH/NICHD (R00HD075858-03 to M.A.S), and the German Research Foundation (Deutsche Forschungsgemeinschaft DFG, SA 2795/2-1 to M.K.K.).

\section{Conflict of Interest}

None declared.

\section{Acknowledgments}

The authors would like to thank Dominique Thompson and Cindy Shope for their work on this project. 


\section{References}

1 Kassi E, Pervanidou P, Kaltsas G, Chrousos G. Metabolic syndrome: definitions and controversies. BMC Med 2011;9:48

2 Shoelson SE, Lee J, Goldfine AB. Inflammation and insulin resistance. J Clin Invest 2006;116(07):1793-1801

3 Kern PA, Ranganathan S, Li C, Wood L, Ranganathan G. Adipose tissue tumor necrosis factor and interleukin- 6 expression in human obesity and insulin resistance. Am J Physiol Endocrinol Metab 2001;280(05):E745-E751

4 Cani PD, Delzenne NM. The role of the gut microbiota in energy metabolism and metabolic disease. Curr Pharm Des 2009;15(13): 1546-1558

5 Cani PD, Amar J, Iglesias MA, et al. Metabolic endotoxemia initiates obesity and insulin resistance. Diabetes 2007;56(07):1761-1772

6 Laugerette F, Vors C, Peretti N, Michalski MC. Complex links between dietary lipids, endogenous endotoxins and metabolic inflammation. Biochimie 2011;93(01):39-45

7 Neves AL, Coelho J, Couto L, Leite-Moreira A, Roncon-Albuquerque R Jr. Metabolic endotoxemia: a molecular link between obesity and cardiovascular risk. J Mol Endocrinol 2013;51(02):R51-R64

8 Hawkesworth S, Moore SE, Fulford AJ, et al. Evidence for metabolic endotoxemia in obese and diabetic Gambian women. Nutr Diabetes 2013;3:e83

9 Benjamin MA, McKay DM, Yang PC, Cameron H, Perdue MH. Glucagon-like peptide-2 enhances intestinal epithelial barrier function of both transcellular and paracellular pathways in the mouse. Gut 2000;47(01):112-119

10 Cani PD, Possemiers S, Van de Wiele T, et al. Changes in gut microbiota control inflammation in obese mice through a mechanism involving GLP-2-driven improvement of gut permeability. Gut 2009;58(08):1091-1103

11 Maruta K, Takajo T, Akiba Y, et al. GLP-2 acutely prevents endotoxin-related increased intestinal paracellular permeability in rats. Dig Dis Sci 2020;65(09):2605-2618

12 Moyce BL, Dolinsky VW. Maternal $\beta$-cell adaptations in pregnancy and placental signalling: implications for gestational diabetes. Int J Mol Sci 2018;19(11):E3467

13 Ernst S, Demirci C, Valle S, Velazquez-Garcia S, Garcia-Ocaña A. Mechanisms in the adaptation of maternal $\beta$-cells during pregnancy. Diabetes Manag (Lond) 2011;1(02):239-248

14 Yogev Y, Catalano PM. Pregnancy and obesity. Obstet Gynecol Clin North Am 2009;36(02):285-300, viii

15 Aviram A, Hod M, Yogev Y. Maternal obesity: implications for pregnancy outcome and long-term risks-a link to maternal nutrition. Int J Gynaecol Obstet 2011;115(Suppl 1):S6-S10

16 Zhu Y, Zhang C. Prevalence of gestational diabetes and risk of progression to type 2 diabetes: a global perspective. Curr Diab Rep 2016;16(01):7

17 Johnson J, Clifton RG, Roberts JM, et al; Eunice Kennedy Shriver National Institute of Child Health and Human Development (NICHD) Maternal-Fetal Medicine Units (MFMU) Network* Pregnancy outcomes with weight gain above or below the 2009 Institute of Medicine guidelines. Obstet Gynecol 2013;121(05):969-975

18 Basu S, Haghiac M, Surace P, et al. Pregravid obesity associates with increased maternal endotoxemia and metabolic inflammation. Obesity (Silver Spring) 2011;19(03):476-482
19 Hanson M, Godfrey KM, Lillycrop KA, Burdge GC, Gluckman PD. Developmental plasticity and developmental origins of noncommunicable disease: theoretical considerations and epigenetic mechanisms. Prog Biophys Mol Biol 2011;106(01):272-280

20 Suter MA, Aagaard-Tillery KM. Environmental influences on epigenetic profiles. Semin Reprod Med 2009;27(05):380-390

21 Antony KM, Hemarajata P, Chen J, et al. Generation and validation of a universal perinatal database and biospecimen repository: PeriBank. J Perinatol 2016;36(11):921-929

22 Chabarria KC, Racusin DA, Antony KM, et al. Marijuana use and its effects in pregnancy. Am J Obstet Gynecol 2016;215(04): 506

23 Kahr MK, Suter MA, Ballas J, et al. Preterm birth and its associations with residence and ambient vehicular traffic exposure. Am J Obstet Gynecol 2016;215(01):111

$24 \mathrm{Kahr}$ MK, Suter MA, Ballas J, et al. Geospatial analysis of food environment demonstrates associations with gestational diabetes. Am J Obstet Gynecol 2016;214(01):110

25 Kahr MK, Antony KM, DelBeccaro M, Hu M, Aagaard KM, Suter MA. Increasing maternal obesity is associated with alterations in both maternal and neonatal thyroid hormone levels. Clin Endocrinol (Oxf) 2016;84(04):551-557

26 American College of Obstetricians and Gynecologists. ACOG Committee opinion no. 548: weight gain during pregnancy. Obstet Gynecol 2013;121(01):210-212

27 Carpenter MW, Coustan DR. Criteria for screening tests for gestational diabetes. Am J Obstet Gynecol 1982;144(07):768-773

28 Mack LR, Tomich PG. Gestational diabetes: diagnosis, classification, and clinical care. Obstet Gynecol Clin North Am 2017;44(02): 207-217

29 Powers R, Kotchey N, Gandley R, Jeyabalan A, Hubel C. PP180. Maternal plasma endotoxin increases significantly across pregnancy with no association with obesity, inflammation, or insulin sensitivity. Pregnancy Hypertens 2012;2(03):337-338

30 Mokkala K, Pellonperä O, Röytiö H, Pussinen P, Rönnemaa T, Laitinen $\mathrm{K}$. Increased intestinal permeability, measured by serum zonulin, is associated with metabolic risk markers in overweight pregnant women. Metabolism 2017;69:43-50

31 Zaretsky MV, Alexander JM, Byrd W, Bawdon RE. Transfer of inflammatory cytokines across the placenta. Obstet Gynecol 2004;103(03):546-550

32 Ben-Hur H, Gurevich P, Elhayany A, Avinoach I, Schneider DF, Zusman I. Transport of maternal immunoglobulins through the human placental barrier in normal pregnancy and during inflammation. Int J Mol Med 2005;16(03):401-407

33 Aagaard K, Ma J, Antony KM, Ganu R, Petrosino J, Versalovic J. The placenta harbors a unique microbiome. Sci Transl Med 2014;6 (237):237ra65

34 Prince AL, Chu DM, Seferovic MD, Antony KM, Ma J, Aagaard KM. The perinatal microbiome and pregnancy: moving beyond the vaginal microbiome. Cold Spring Harb Perspect Med 2015;5(06): a023051

35 Antony KM, Ma J, Mitchell KB, Racusin DA, Versalovic J, Aagaard K. The preterm placental microbiome varies in association with excess maternal gestational weight gain. Am J Obstet Gynecol 2015;212(05):653 